\title{
Recent Investigations of Hydromagnetic Emissions Part II. Theoretical Interpretation
}

\author{
R.C. WENTWORTH \\ Research Laboratories \\ Lockheed Missiles and Space Company, Palo Alto, California
}

\begin{abstract}
Recent experimental and theoretical work suggests the hypothesis that hydromagnetic (hm) emissions are hydromagnetic wave packets guided by field lines as they bounce back and forth between hemispheres of the earth. As such they are analogous to whistlers, and one test of the hypothesis is the quantitative calculation of plasma densities in the outer magnetosphere and their comparison with whistler densities closer to the earth.

Many hm emission exhibit measurable dispersion when displayed in the form of sonagrams (frequency vs time displays). In such cases, the measurement of group bounce times at two different emission frequencies can be combined with an accurate model of the magnetosphere to determine the zero-frequency (Alfvén) bounce period and the equatorial cyclotron frequency above which the wave cannot propagate. These two quantities uniquely define the field line along which the hm emission propagated and the integrated plasma density along that field line.

Analysis of 9 events showed they occurred on field lines crossing the equatorial plane between 4 and 10 earth radii, and showed equatorial plasma densities in agreement with extrapolated whistler "knee" densities.
\end{abstract}

\section{Introduction}

The existence of distinctive quasi-sinusoidal low-amplitude oscillations of the earth's magnetic field in a limited frequency interval around $1 \mathrm{cps}$ has been recognized for some time (Troitskaya, 1957; Duffus et al., 1958; Benioff, 1960), but until very recently practically nothing was known about their origin. However, in the past few years advances in experimental technique have revealed enough new properties of these signals, called "pearls" by Troitskaya and hydromagnetic $(\mathrm{hm})$ emissions by Tepley (1961), to enable the construction of quantitative models of their generation and propagatio propagation.

The most important improvement in technique was the recording of these signals on magnetic tape, making possible their reduction to the form of sonagrams (frequency versus time displays). This led to the discovery that many $\mathrm{hm}$ emissions are characterized by a remarkable frequency-time fine structure (Tepley, 1961, 1962; 1964; Gendrin and Stefant, $1962 \mathrm{a}, \mathrm{b}$; Mainstone and McNicol, 1962; Tepley and Wentworth, $1962 \mathrm{a}, \mathrm{b}, 1963$; Tepley et al., 1964. experimental reviews have been written by Jacobs and Watanabe, 1963a; Campbell, 1964; Tepley, 1964, 1966, hereafter called Part I). In particular, hm emissions consisting of a band of frequencies (typically $\Delta f / f_{c} \approx 0.5$ where $\Delta f$ is the bandwidth and $f_{c}$ is the center 
can usually be interpreted as a series of repetitive overlapping wave trains of rapidly rising frequency (typically $\mathrm{d} f / \mathrm{d} t$ varies between 0.1 and $0.5 \mathrm{cps} / \mathrm{min}$; Tepley and Wentworth, 1962b). Examples of such spectra are shown in Part I, Figures 2 and 3.

In addition, recent experimental observations (Tepley, 1964; Tepley et al., 1964) have conclusively demonstrated that whereas the regularly spaced fine-structured elements which constitute most $\mathrm{hm}$ emissions occur within several seconds of each other at stations in the same hemisphere, they are alternately spaced $\left(180^{\circ}\right.$ out of phase) for stations on opposite sides of the equator (Part I, Figure 10). Hence, hm-emission energy is received periodically and alternately in the northern and southern hemispheres. This result is suggestive of a slow energy bounce between hemispheres (bounce times are typically on the order of 1 to 5 minutes).

The demonstration of the $180^{\circ}$ phase shift eliminated as untenable a number of "fast" charged-particle bunch models for the generation of $\mathrm{hm}$ emissions which predicted $0^{\circ}$ phase shifts between hemispheres (Wentworth and Tepley, 1962; Heacock, 1963; Gendrin, 1963a, b, c). This left as reasonable possibilities only the slow proton bunch model proposed by a number of authors (Yanagihara, 1959, 1963; Watanabe, 1959; Obayashi, 1959; Jacobs and Watanabe, 1962, 1963b; Lokken et al., 1963) and the hydromagnetic wave model proposed by Obayashi (1964), and Jacobs and Watanabe (1964a).

In the slow proton bunch model of Jacobs and Watanabe (1963b) the emission frequency is associated with the resonance frequency of hydromagnetic waves generated in the lower exosphere (Jacobs and Watanabe, (1962). The resonance oscillations are supposedly excited by proton bunches oscillating slowly between magnetically conjugate mirror points. The fine-structure periodicity is associated with the proton bunch bounce period. Consequently the model correctly predicts the observed $180^{\circ}$ conjugatehemisphere fine-structure phase shift. The rising-frequency fine structure is attributed to a latitude variation of the properties of the lower exosphere, which give rise to the hydromagnetic resonance effect. However, a serious drawback is that the model predicts that the emission frequency should increase with geomagnetic latitude, a prediction in conflict with experimental observations (Tepley and Wentworth, 1962b; Heacock and Hessler, 1962; Gendrin, 1963b, his Figure 1; Matveeva and Troitskaya, 1963; Wentworth, 1964). Since this model also seems unlikely to be responsible for the generation of hm emissions, it will no longer be considered in the present paper.

An alternative model consistent with the $180^{\circ}$ phase shift is the slow bounce of a hydromagnetic wave packet guided by magnetic field lines between hemispheres. Jacobs and Watanabe (1964a) presented a model in which hm emissions observed at sea level are the boundary effects of hydromagnetic wave packets bouncing between hemispheres. The wave packets propagate in the left-hand polarized Alfvén mode and are partially guided by the geomagnetic field in much the same way as whistlers (Storey, 1953). Jacobs and Watanabe show that a hydromagnetic wave packet propagating along a magnetic line of force of the earth's field will be dispersed into a sequence of rising tones. Their calculations are in close agreement with the experimentally observed dynamic spectra of most hm emissions (cf Part I, Figures 2 and 3). However, it should be pointed out that this model for hm emis- 
sions is not complete. This is because $\mathrm{hm}$ emissions are frequently observed to have a gradual increase in amplitude of successive wave packets (typically over a 10 to 15 minute period, Part I, Figure 1) which implies that energy is being continuously supplied to the wave packet.

The problem of the gradual increase in amplitude of successive wave packets has been considered in a number of very recent models (Jacobs and Watanabe, 1964b, 1965; Obayashi, 1964; Tepley and Wentworth, 1964; Cornwall, 1965) which attribute hm emissions to either Doppler shifted cyclotron radiation or Cerenkov radiation from charged particles in the magnetosphere. In an elegant treatment, Cornwall (1965) considers a particular instability that can take place when a beam of fast protons passes through a cold plasma imbedded in a uniform field. He shows that under certain conditions the instability takes place at cyclotron resonance-that is, when the Doppler-shifted wave frequency is equal to the cyclotron frequency. This instability is the exact analog, for protons, of a cyclotron instability discussed by Dawson and Bernstein (1958) for a beam of electrons. Thus a source of energy to account for the gradual increase in amplitude of successive wave packets would exist if protons having a specific distribution function were available in the magnetosphere.

Thus the bouncing-wave-packet model of Jacobs and Watanabe is a reasonable hypothesis for the propagation of $\mathrm{hm}$ emissions in the magnetosphere. It is therefore desirable to examine this hypothesis quantitatively; one test is that plasma densities in the outer magnetosphere (from 5 to 10 earth radii as it turns out) can be derived from observed hm emission dispersion and compared with extrapolated whistler densities.

In order to make such a quantitative calculation it is desirable to work with a quantitative model of the magnetic field in the magnetosphere. Since field lines along which the wave packets propagate cross the equatorial plane from 4 to 10 earth radii they are significantly distorted by external currents flowing on the surface and in the tail of the magnetosphere. It is therefore not sufficiently accurate to employ a pure dipole field model, and it is necessary to estimate the effects of the perturbing currents. Since it is likely that the currents flowing on the front surface of the magnetosphere induced by the solar wind produce the most significant distortions in the sub-solar region the noon-meridian field calculated by Mead (1964) for the interior of a model magnetosphere cut off at 10 earth radii has been employed. The situation in the antisolar portion of the magnetosphere is more speculative. However, for the Durpose of the calculations it was assumed that the currents flowing in the neutral sheet (Ness, 1964) formed the principal distortion of the field, and that their effect could be approximated by subtracting from a pure dipole field a uniform field equal to one-half the pure dipole field at 8 earth radii (inner boundary of the neutral sheet, Ness, private communication). These field lines are shown in Figure 1 where it can be seen that the field is compressed on the sun side of the magnetosphere, and is elongated and separated into a connected and non-connected region on the anti-solar side.

In addition it was necessary to assume the form of the distribution of plasma along a line of force, and for the purpose of these calculations it was assumed that the density was proportional to $r^{-3}$ (cf. Liemohn and Scarf, 1964), where $r$ is the distance from the center of 


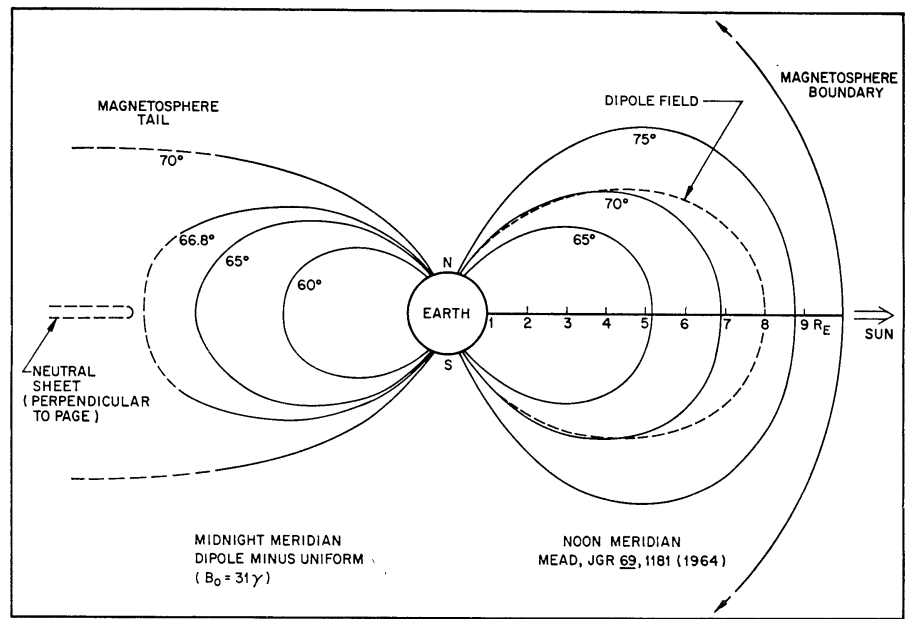

Fig. 1 Magnetic field model of the magnetosphere. The noon-meridian field lines are those given by Mead (1964) for a magnetosphere cut off at 10 earth radii by the solar wind. Note that they are compressed relative to the dotted dipole field line. The midnight-meridian field lines have been calculated by subtracting from a pure dipole field a uniform vertical field of 31 gammas. This is approximately the effect produced by a discontinuous diamagnetic ring current with a sharp inner edge at 8 earth radii. As discussed in the text it is assumed that the neutral sheet current system has this approximate effect and location. The resultant field lines are drawn out relative to pure dipole field lines. The present model is probably not accurate too close to the neutral sheet, so that field lines beyond 7 earth radii have been sketched in as dotted lines.

the earth.

Analysis of 6 nighttime and 3 daytime events (as explained in the text) showed they occurred on field lines between 4 and 10 earth radii (half the events occurred on field lines beyond 5.75 earth radii) and showed equatorial plasma densities in good agreement with the "knee" densities beyond 3 to 4 earth radii inferred from whistler data (Carpenter, 1963). Thus since hm-emission events "probe" the outer portions of the "connected" magnetosphere from 4 to 10 earth radii with some regularity they are a very valuable source of information on this extremely interesting portion of the magnetosphere.

\section{Dispersion Relation}

Propagation of the electromagnetic radiation discussed in the present paper in the magnetosphere will be described by a brief discussion of the dispersion relation as derived Stix (1962) in the cold-plasma approximation. Our medium is assumed to be a two-component plasma of protons and electrons at very low temperature. We are interested in conditions such that the following inequalities are satisfied:

$$
\omega \lesssim \Omega_{i} \ll \Omega_{e} ; m_{i} \gg m_{e}
$$




$$
r=\frac{4 \pi n m_{i} c^{2}}{B_{0}{ }^{2}}=\frac{c^{2}}{v_{A}{ }^{2}} \gg 1
$$

where $\omega$ is the wave frequency, $\Omega_{i}$ is the ion cyclotron frequency, and $v_{A}$ is the Alfven velocity. In general, the notation in this section will be that employed by Stix.

We define $\theta$ as the angle between the direction of the propagation vector and the static magnetic field, and under the restriction that $\theta$ is not close to $90^{\circ}$,

$$
\left(\frac{\Omega_{i}}{\omega}\right)^{2}-1 \gg \frac{\Omega_{i}}{\Omega_{e}} \tan ^{2} \theta
$$

the dispersion equation becomes (Stix, Equation 2-28)

$$
n^{4} \cos ^{2} \theta-n^{2} \frac{r \Omega_{i}{ }^{2}}{\Omega_{i}{ }^{2}-\omega^{2}}\left(1+\cos ^{2} \theta\right)+\frac{r^{2} \Omega_{i}{ }^{2}}{\Omega_{i}{ }^{2}-\omega^{2}}=0
$$

The solutions to this equation are given by

$$
k^{2} c^{2} / \omega^{2} \equiv n_{ \pm}{ }^{2}=\frac{r \Omega_{i}{ }^{2}}{2 \cos ^{2} \theta\left(\Omega_{i}{ }^{2}-\omega^{2}\right)}\left\{\left(1+\cos ^{2} \theta\right) \pm\left[\sin ^{4} \theta+4 \cos ^{2} \theta\left(\frac{\omega}{\Omega_{i}}\right)^{2}\right]^{1 / 2}\right\}
$$

In order to define the two modes represented by the two solutions of Equation 2, we let $\theta=0^{\circ}$. Then

$$
\begin{aligned}
& n_{+}{ }^{2}=\frac{r \Omega_{i}}{\Omega_{i}-\omega}=L \\
& n_{-}{ }^{2}=\frac{r \Omega_{i}}{\Omega_{i}+\omega}=R
\end{aligned}
$$

Stix shows in his Equation 1-31 that for $\theta=0^{\circ}$ the polarization is circular with a right- or left-hand sense according to $n^{2}=R$ or $n^{2}=L$, respectively. Therefore, the solution $n_{+}{ }^{2}$ refers to the ion cyclotron wave which is left. hand circularly polarized for $\theta=0^{\circ}$.

In the zero-frequency approximation (with $\theta$ not too close to $0^{\circ}$ ),

$$
\frac{\omega}{\Omega_{i}} \ll \frac{\sin ^{2} \theta}{2 \cos \theta}
$$

Equation 2 reduces to

$$
n_{+}^{2}=\frac{r}{\cos ^{2} \theta} ; n_{-}^{2}=r
$$

As discussed by Stix (Equations 3-18 and 3-19, and Figures 3--2 and 3-3) the direction of energy flow in a loss free medium, called the ray direction, is along the group-velocity vector. The group velocity vector, $\boldsymbol{v}_{g}$, is given by

$$
\boldsymbol{v}_{g}=\boldsymbol{i} \frac{\partial \omega}{\partial k_{x}}+\boldsymbol{j} \frac{\partial \omega}{\partial k_{y}}+\boldsymbol{k} \frac{\partial \omega}{\partial k_{z}}
$$

Therefore, if we can find a coordinate system for which

$$
\frac{\partial \omega}{\partial k_{y}}=\frac{\partial \omega}{\partial k_{z}}=0
$$


the group velocity will be perpendicular to the $y-z$ plane. However, just such a coordinate system exists on a set of curves called inverse wave normal surfaces (Stix, 1962, p 53) or surfaces of refractive index (Jacobs and Watanabe, 1964). These curves are surfaces of revolution generated by rotating $n(\theta)$ in Equation 2 (plotted in polar coordinates with $\theta$ measured from Bo) about the direction of the static magnetic field Bo. Since by definition $\omega$ is constant on a surface of refractive index Equation 5 will be satisfied everywhere in the surface and the group velocity vector will be everywhere perpendicular to the surface.

The $n(\theta)$ surfaces for a particular plasma density and magnetic field strength appropriate to the outer magnetosphere have been plotted by Jacobs and Watanabe (1964). These surfaces show that the group velocity vector is confined within a very small cone centered about the direction of the static magnetic field for the ion cyclotron wave, and is not significantly restricted by the direction of the static magnetic field for the "fast" mode. We note in particular from Equation 4 that in the zero-frequency limit the $n(\theta)$ surface for the ion cyclotron wave is a plane perpendicular to the magnetic field direction, so that energy is guided completely along the magnetic field direction in this case.

It is of interest, for the sake of completeness, to discuss briefly the analogous situation for whistlers. Storey (1953) showed that in the low-frequency limit the dispersion relation reduced to

$$
n^{2}{ }_{w h i s t l e r} \approx \frac{a^{2}}{\cos \theta}
$$

If we let

$$
y=n \cos \theta, \quad x=n \sin \theta
$$

we obtain

$$
\cot \psi=\frac{d x}{d y}=-\frac{x}{y}\left[\frac{a^{4}+y^{4}}{a^{4}-y^{4}}\right]
$$

where $\psi$ is the angle between the group velocity vector (perpendicular to the $n(\theta)$ curve) and the magnetic field. This function has a minium at

$$
y^{4}=\frac{1}{3} a^{4}
$$

so that the maximum value of $\Psi$ is given by

$$
\begin{aligned}
& \cot \Psi_{\max }=8^{1 / 2} \\
& \Psi_{\text {max }}=19^{0} 58^{\prime}
\end{aligned}
$$

This result derived by Storey shows that whistler mode radiation in the low-frequency limit is confined within a cone $19^{\circ} 28^{1}$ wide centered about the magnetic field direction.

It thus appears on the basis of Equations 4 and 6 that in the zero-Constrained frequency approximation the ion cyclotron wave is more completely constrained to propagate along the magnetic field than is the whistler mode VLF wave. It is thus likely that hydromagnetic emissions propagate along field lines in the magnetosphere in the left-hand circularly pola- 
rized ion cyclotron mode. However, it will be noted that sinu considerable dispersion is often observed the zero-frequency approximation is probably not valid for $\mathrm{hm}$ emissions in the outer magnetosphere, and the question of the guidance of these signals is at present unsolved.

\section{Hydromagnetic Wave Propagation}

1. Alfvén wave bounce period. In order to calculate transit times for Alfvén waves in the magnetosphere it is first necessary to calculate the Alfvén velocity, $v_{A}$, given by

$$
V_{A}=B / \sqrt{4 \pi \rho m_{i}}
$$

The magnetosphere field model used in the the present calculations is described in the Appendix and plotted in Figures 1-4. Then, substitution of Equations A4 and A5 into Equation 8 yields the equatorial Alfvén velocity curves plotted in Figulre 5 where they are compared with the recent Dessler (1965) model.

The bounce period $T_{A}$ for a wave having the Alfvén velocity to travel along a line of force from one hemisphere to the other and return has been calculated from Equations A4 and 8 and Figure 4. The result has been plotted in Figure 6. We thus have for the Alfvén bounce period $T_{A}$,

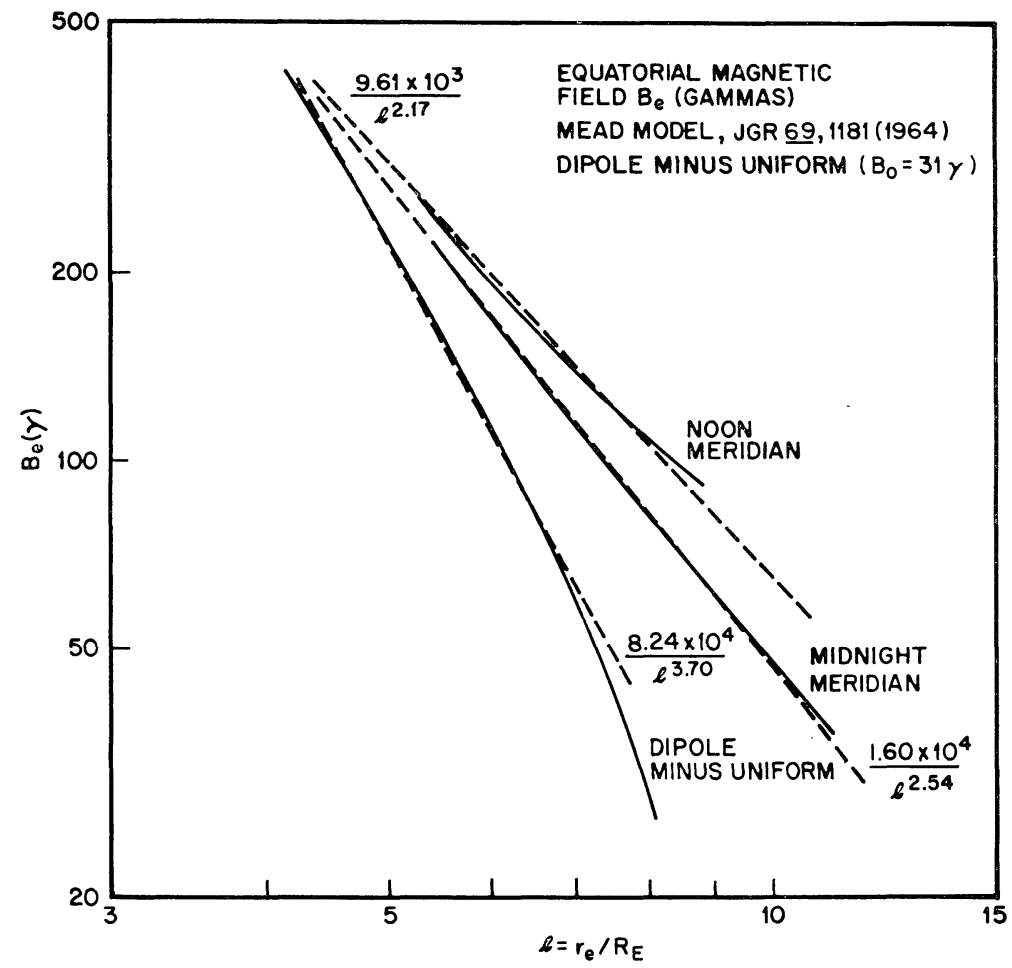

Fig. 2 Equatorial magnetic field strengths. The model used in the present calculations consists of the Mead (1964) noon-meridian curve and the dipole-minus-uniform curve for the anti-solar magnetosphere. The Mead midnight-meridian curve has been included for the sake of comparison. 


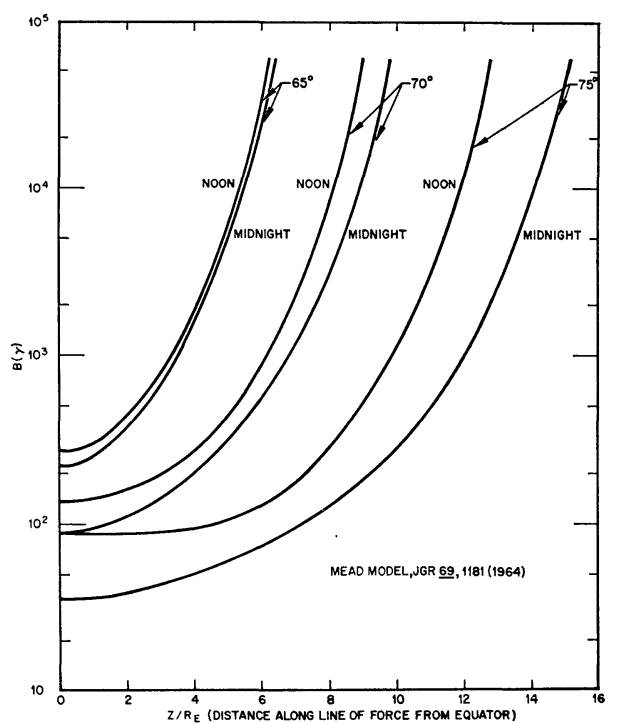

Fig. 3 Magnetic field strengths as a function of distance along the line of force for the Mead (1964) noon- and midightmeridian field lines reaching the earth at $65^{\circ}, 70^{\circ}$, and $75^{\circ}$ latitude.

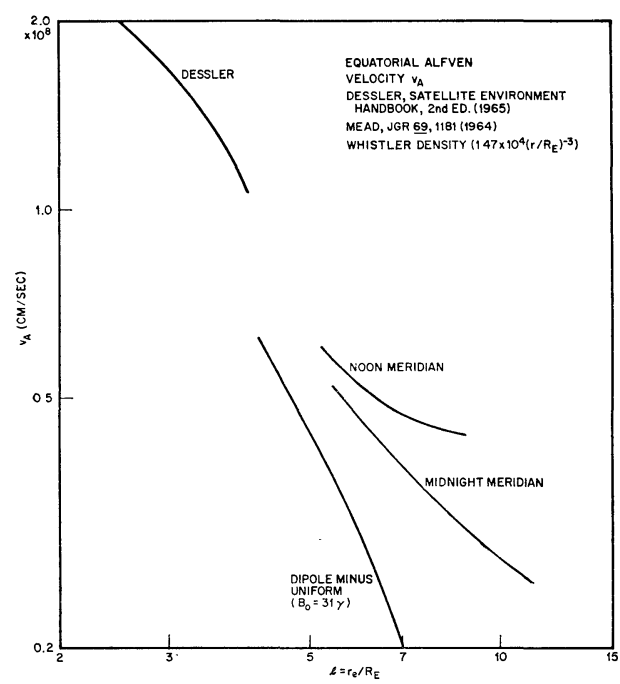

Fig. 5 Equatorial Alfvén velocities for the Mead (1964) noon- and midnight-meridian models and the dipole-minusuniform model. A standard whistler density has been assumed, and these curves compare well with the Dessler (1965) values closer to the earth.

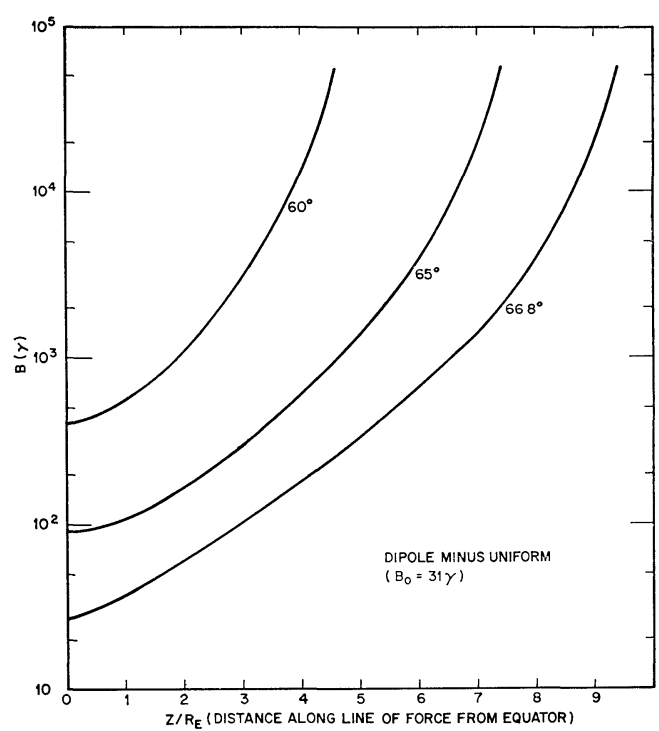

Fig. 4 Magnetic field strengths as a function of distance along the line of force for the dipole-minus-uniform field lines reaching the earth at $60^{\circ}, 65^{\circ}$, and $66.8^{\circ}$ latitude.

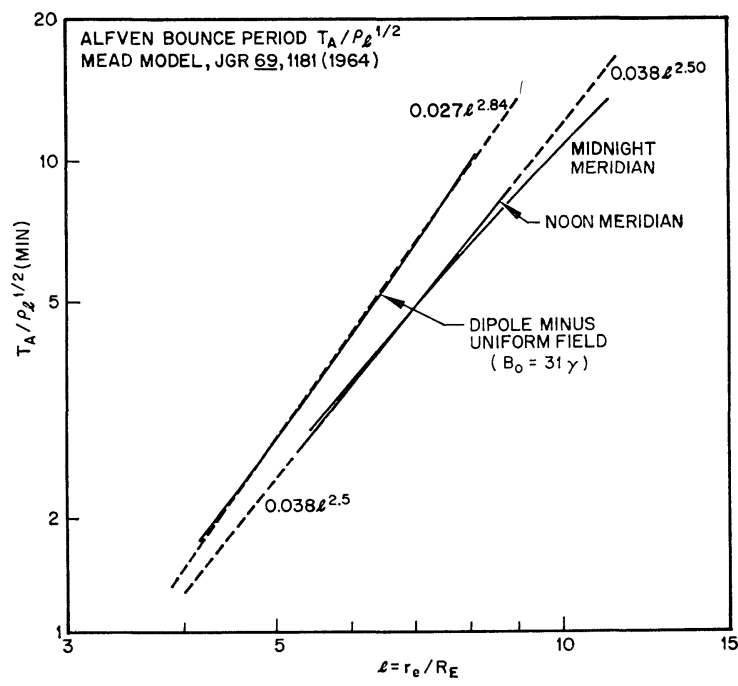

Fig. 6 The Alfvén bounce period as a function of equatorial crossing distance for the Mead (1964) noon- and midnight-meridian models and the dipole-minus-uniform model. These curves have been calculated for a standard whistler density. 


$$
\left.\begin{array}{ll}
T_{A}=0.038 \rho_{l}^{1 / 2} l^{2.50} & \text { (noon) } \\
T_{A}=0.027 \rho_{l}^{1 / 2} l^{2.84} & \text { (midnight) }
\end{array}\right\} \text { minutes }
$$

2. Dispersion of $h m$ emissions: "group" bounce period. The group velocity, $v_{g}$, is obtained for the ion cyclotron wave propagating parallel to the magnetic field from Equation 5 ,

$$
v_{g}=\frac{\partial \omega}{\partial k}=v_{A} \frac{(1-\zeta)^{3 / 2}}{(1-\zeta / 2)}
$$

where $\zeta=f / f_{c}$ is the ratio between the wave frequency and the local proton cyclotron frequency, which is given by

$$
f_{c}=1.525 \times 10^{3} B \text { cps }(B \text { in gauss })
$$

It should be emphasized that we have chosen to work with the ion-cyclotron wave which is left-hand circularly polarized for propagation along the field line since in the zero-frequency limit the wave energy is propagated only along the direction of the eternal magnetic field. Thus, as first pointed out by Jacobs and Watanabe (1964) the ion-cyclotron wave will be partially guided by field lines in the magnetosphere in much the same way as whistlers, and the problem of ducting is much less severe than it would be for the "fast"

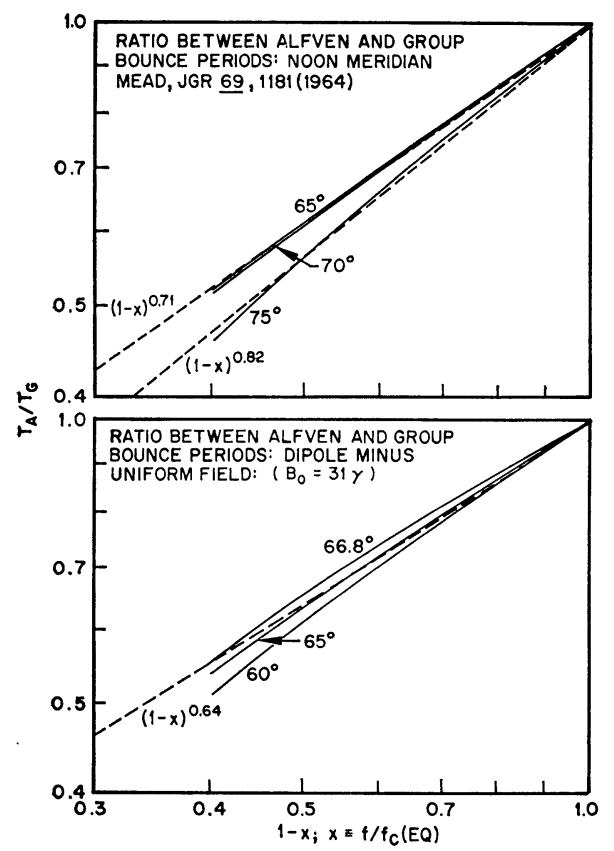

Fig. 7 Ratio between Alfvén and "group" bounce periods for the Mead (1964) noon-meridian model and for the dipole-minus-uniform model as a function of 1 minus the ratio between the wave frequency and the equatorial proton cyclotron frequency. mode. In addition, as pointed out by a number of authors (Jacobs and Watanabe, 1964b, 1965; Obayashi, 1964; Tepley and Wentworth, 1964; Cornwall, 1965) instabilities exist which take place at cyclotron resonance in which the left-hand circularly polarized mode can be amplified by protons of moderate energies in the magnetosphere.

It is a simple matter to calculate group bounce periods from Equation 10, and the results are plotted in Figure 7. If we define $x$ as the ratio between the wave frequency and the minium proton cyclotron frequency along the line of force (according to Mead, noon-meridian field lines above $75^{\circ}$ have their minimum field strength off theequatorial plane) then the ratio between $T_{A}$ and $T_{g}$ is accurately represented by a power low in $1-x$,

$$
T_{A} / T_{g}=(1-x)^{b}
$$

where

$$
x \equiv f / f_{c}(\min ) \quad\left(f_{c}(\min )=f_{c}(\mathrm{lc}), \lambda_{E} \leq 75^{\circ}\right)
$$




$$
b= \begin{cases}0.71\left(65^{0}, 70^{\circ}\right) & \} \text { noon } \\ 0.82\left(75^{0}\right) & \text { midnight } \\ 0.64\left(B_{0}=31 \gamma\right) & \end{cases}
$$

Equation 12 contains two unknown quantities, $T_{A}$, and $f_{c}$. The quantities which are experimentally determined are the wave frequency $f$ and the group bounce period $T_{g}$ (finestructure repetition period in Part I, Figure 3). Therefore, under the assumption that a single wave packet containing a band of frequencies is propagating along a single line of force, the two unknowns, $T_{A}$ and $f_{c}$, may be determined in a given event by measuring two sets of values of $T_{g}$ and $f$. If we measure the sets of values $T_{1}$ and $f_{1}$, and $T_{2}$ and $f_{2}$, we have from Equation 12,

$$
\frac{f_{r}}{f_{1}}=\frac{\left(f^{2} / f_{1}\right)\left(T_{2} / T_{1}\right)^{1 / b}-1}{\left(T_{2} T_{1}\right)^{1 / b}-1}
$$

and

$$
T_{A}=T_{1}\left(1-f_{1} / f_{c}\right)^{b}
$$

3. Guiding line of force. The line of force along which an observed $\mathrm{hm}$ emission is bouncing is uniquely determined from the equatorial proton cyclotron frequency calculated in Equation 13 (field lines above $75^{\circ}$ in the noon meridian have not been considered here). The line of force is specified by its equatorial crossing distance from the center of the earth. From Equations A4 and 11 we have

$$
\left.\begin{array}{ll}
l=\left(147 / f_{c}\right)^{0.46} & \text { (noon) } \\
l=\left(1256 / f_{c}\right)^{0.27} & \text { (midnight) }
\end{array}\right\}
$$

4. Equatorial exospheric density. Once the field line has been determined from Equation 15, the Alfvén bounce period calculated in Equation 14 uniquely determines the equatorial plasma density. Thus using Equations A5 and 9, we have for the equatorial plasma density,

$$
\rho_{l}=1.02 \times 10^{7} T_{A}^{2} l^{-8}
$$

Table 1 Dispersion measured for $9 \mathrm{hm}$-emission events. Also shown is the plasma density derived by the present model calulations.

\begin{tabular}{c|ccccccc|l}
\hline Date & GMT & $f_{1}(\mathrm{cps})$ & $T_{1}(\min )$ & $f_{2}(\mathrm{cps})$ & $T_{2}(\min )$ & $l$ & $\rho\left(\mathrm{cm}^{-3}\right)$ & \\
\hline 13 Jul 61 & 1207 & 1.40 & 0.77 & 2.35 & 1.46 & 5.14 & 3.53 & \\
02 Nov 61 & 0800 & 1.30 & 1.35 & 1.80 & 1.57 & 4.81 & 26.4 & \\
26 Dec 62 & 1130 & 1.04 & 1.68 & 1.66 & 1.96 & 4.75 & 51.0 & Midniht \\
04 Feb 63 & 0615 & 1.01 & 1.97 & 1.20 & 2.28 & 5.75 & 7.52 & Meridian \\
04 Feb 63 & 1250 & 1.11 & 1.92 & 1.38 & 2.07 & 4.97 & 40.0 & \\
10 Apr 63 & 0645 & 0.33 & 2.52 & 0.47 & 3.28 & 7.41 & 0.17 & \\
\hline 05 Feb 63 & 1900 & 0.77 & 2.23 & 1.00 & 2.99 & 8.16 & 0.81 & Noon \\
21 Feb 63 & 1530 & 0.47 & 2.23 & 0.59 & 2.55 & 8.95 & 0.57 & Meridian \\
01 Apr 63 & 1930 & 0.39 & 2.53 & 0.45 & 2.61 & 7.22 & 6.38 & \\
\hline
\end{tabular}




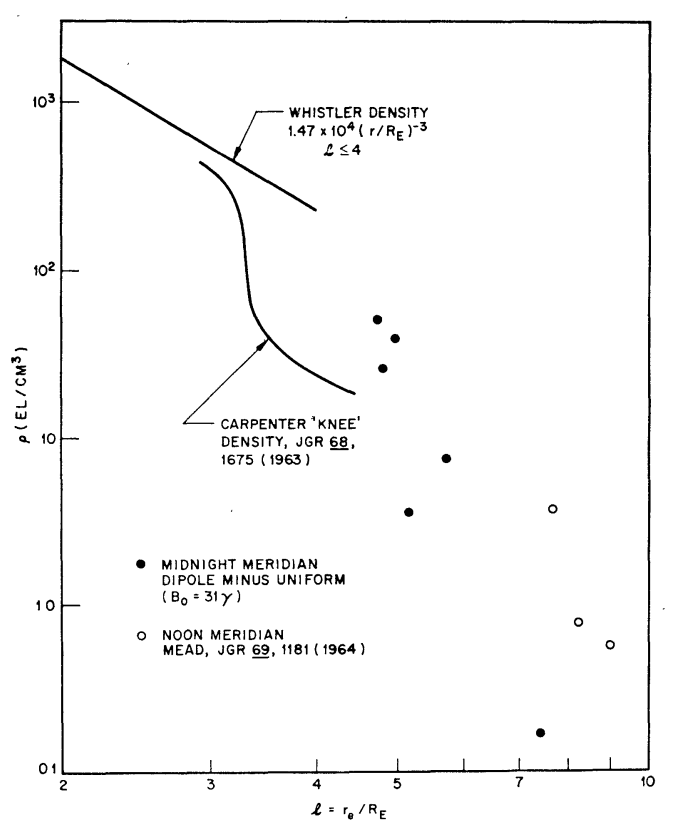

Fig. 8 Equatorial plasma densities derived from $\mathrm{hm}$-emission dispersion data for nine events. The events represented by the solid dots occurred during local night, and the dipoleminus-uniform field model was used in calculating them. The events represented by the open circles occurred during local day, and the Mead (1964) noon-meridian field was used in their calculation. It can be seen that the plasma densities derived from these calculations lie well below the standard whistler curve extrapolated beyond 4 earth radii, but are in good agreement in extending the "knee" densities discovered by Carpenter (1963).

\section{Experimental Results}

Nine hm-emission events including that of Part I, Figure 3 have been analyzed according to the technique discussed in the earlier sections. The dispersion measured for these events is presented in Table 1 , and the equatorial plasma densities derived from them have been plotted in Figure 8. It can be seen that the results are in good agreement with extrapolated whistler "knee" densities as given by Carpenter (1963).

\section{Conclusions}

Very recent models of $\mathrm{hm}$ emissions are based on the hypothesis that they are hydromagnetic wave packets bouncing between hemispheres along magnetic field lines which lie in the outer magnetosphere. In order to test this hypothesis quantitatively calculations have been performed which derive plasma densities along the field lines analogous to whistler applications. The results are extremely promising in showing densities consistent with extrapolated "knee" densities from whistler data (Carpenter, 1963). Therefore, the model hypothesis that hm emissions are hydromagnetic wave packets propagating along magnetic field lines in the left-hand circularly polarized ion cyclotron mode is strongly svpported.

In addition, based on a limited data sample, it is concluded that plasma densities in the outer magnetosphere are low, varying from roughly $50 / \mathrm{cm}^{3}$ at 5 earth radii to $0.5 / \mathrm{cm}^{3}$ at 9 earth radii.

\section{Appendix}

1. Magnetosphere field model. In order to make quantitative calculations of $\mathrm{hm}$ emission bounce times it is desirable to work with the most accurate available model of the 
field in the magnetosphere. It is shown in Table 1 that the field lines along which the wave packets propagate cross the equatorial plane from 4 to 10 earth radii from the center of the earth. Since they are significantly distorted in this region of space by external currents flowing on the surface and in the tail of the magnetosphere it is nəcessary to modify the usual pure dipole model geometry.

It is likely that the currents flowing on the front surface of the magnetosphere induced by the solar wind produce the most significant distortions in the sub-solar hemisphere. Therefore, the noon-meridian field calculated by Mead (1964) for the interior of a model magnetosphere cut off at 10 earth radii by the solar wind has been employed in the present calculations.

In the anti-solar hemisphere the situation is more speculative. However, it is likely that the currents flowing in the neutral sheet (Ness, 1965) produce the principal distortions of the field in this region. These currents may be produced by solar wind particles which are injected on one side of the magnetosphere and then drift across the tail to be lost on the other side. The protons drift from east to west, and the electrons from west to east, the net effect being a westward current system in the equatorial plane similar to a portion of the classical diamagnetic ring current postulated to be responsible for the main phase of magnetic storms (Singer, 1957; Dessler and Parker, 1959). In fact, the possibility exists that the main phase of magnetic storms is solely due to enhanced neutral sheet currents flowing closer to the earth (Piddington, 1960).

Therefore, it is likely that the currents flowing in the neutral sheet produce a perturbation field close to the earth similar in character to that of a diamagnetic ring current. However, Apel (1961), Akasofu and Chapman (1961) and Apel et al. (1962) showed that a diamagnetic ring current produces a perturbation field in the equatorial plane which is nearly constant from the earth out to the position of the current. Such a perturbation field is perpendicular to the equatorial plane by symmetry. In addition, the radius of curvature $\rho_{c}$ of a perturbation field line is definite as it crosses the equatorial plane $\left(\rho_{c}=B / \nabla B\right.$ and $\nabla B$ is approximately zero). Therefore, to a first approximation, an extrapolation off the equatorial plane has the solution that the perturbation field in space near the earth is also uniform and constant. This effect can be seen in Akasofu et al. (1961, Figure 1).

We therefore assume that the midnight-meridian field line is that of a pure dipole with a uniform field $B_{0}$ subtracted from it. The $B_{r}$ and $B_{\lambda}$ components become

$$
\begin{aligned}
& B_{r}=-\left(\frac{2 M}{r^{3}}+B_{0}\right) \sin \lambda \\
& B_{\lambda}=\left(\frac{M}{r^{3}}-B_{0}\right) \cos \lambda
\end{aligned}
$$

and the equation of a line of force from the surface of the earth $\left(R_{E}, \lambda_{E}\right)$ becomes

$$
\left(\frac{\cos \lambda}{\cos \lambda_{E}}\right)^{2}=\frac{r}{R_{E}} \frac{\left(1+B_{0} R_{E}^{3} / 2 M\right)}{\left(1+B_{0} r^{3} / 2 M\right)}
$$


We must now estimate the value $B_{0}$ of the uniform field to be subtracted from the dipole field of the earth. Since the neutral sheet current system is assumed to be composed of quasi-trapped solar wind particles from interplanetary space it must have an inner edge outside the stable trapping region in the magnetosphere. The number density of drifting solar wind particles must decrease abruptly to zero at this inner edge which divides the magnetosphere into connected and unconnected magnetic field geometries. This discontinuity in number density has the interesting effect of producing an eastward current spike, similar to the eastward current system on the subsolar boundary of the magnetosphere, which partially cancels the decrease in the field produced by the neutral sheet as a whole. As can be seen in Apel et al. (1962, Figure 6) the constant negative perturbation field produced by such a discontinuous diamagnetic ring current near the earth is about half the perturbation field produced just within the diamagnetic plasma itself. Therefore, we postulate that the constant field will be equal to one-half the perturbation field produced by the neutral sheet at its innermost edge which is just equal and opposite to the pure dipole field value at that point. We thus have for $B_{0}$,

$$
B_{0}=M / 2 r_{N}^{3}
$$

where $r_{N}$ is the distance from the center of the earth to the inner edge of the neutral sheet.

Disturbance fields in the tail of the magnetosphere which were undoubtedly produced by the currents flowing in the neutral sheet were first observed on Explorer VI and Explorer X. Smith (1962) discusses the two sets of measurements, and plots disturbance fields for Explorer VI of approximately 60 gammas (mildly disturbed conditions) and for Explorer $\mathrm{X}$ of approximately 30 gammas (magnetically quiet). If these are taken to be representative of $B_{0}$ in Equation $\mathrm{A} 3$, then the inner edge of the neutral sheet was located at approximately 6.4 and 8 earth radii, respectively. For the purpose of the present calculations magnetically quiet conditions are assumed, and the inner edge of the neutral sheet is assumed to be at 8 earth radii. This is in agreement with an estimate by Ness (private communication).

Additional evidence for this geometry-connected fields confined to a smaller region in the anti-solar magnetosphere as shown in Figure 1-has been supplied by polar orbiting satellites which show the high-latitude boundary of the stable trapping region to be lower at night (O'Brien, 1963; Williams and Palmer, 1965). Also, indirect evidence for shorter field lines at night is contained in the data published by Heacock and Hessler (1962) which show a median bounce period of $3.4 \mathrm{~min}$ for the hm-emission events which occurred during the 8 hours around local noon at College, Alaska, compared with a median bounce period of $2.6 \mathrm{~min}$ for those events which occurred around local midnight.

The magnetic field lines in the noon- and midnight-meridians are shown in Figure 1. It can be seen that compared to dipole field lines they are compressed in the noon meridian and stretched out in the midnight meridian. The magnetic field strength in the equatorial plane is plotted in Figure 2. It can be seen that the two cases are accurately represented by power laws, so that we have for the noon and midnight equatorial field values 


$$
\left.\begin{array}{ll}
B_{l}=9.61 \times 10^{3} l^{-2.17} & \text { (noon) } \\
B_{l}=8.24 \times 10^{4} l^{-3.70} & \text { (midnight) }
\end{array}\right\} r
$$

where

$$
l \equiv r_{l} / R_{E} ; R_{E}=6.37 \times 10^{8} \mathrm{~cm}
$$

We have included the Mead (1964) midnight-meridian field values for the sake of comparison with the dipole-minus-uniform field model. Finally, for the purpose of our present calcula: tions, we have plotted in Figures 3 and 4 the magnetic field strength as a function of distance along the line of force from the equatorial plane.

2. Exospheric density distribution along a field line. It is assumed that the density along a field line varies proportional to $r^{-3}$, where $r$ is the distance from the center of the earth. We thus have for the density $\rho$ in the magnetosphere,

$$
\rho=1.47 \times 10^{4} \rho_{l}\left(r / R_{E}\right)^{-3} \mathrm{el} / \mathrm{cm}^{3}
$$

In this formulation, $\rho_{l}$ is the dimensionless unknown to be determined by the analysis. According to Liemohn and Scarf (1964) the "best" whistler determined exospheric density during geomagneticelly quiet times would have $\rho_{l}$ equal to unity.

\section{Acknowledgements}

The experimental data used in the present calculations were obtained by Lockheed under partial support by the following government agencies: 1) AFCRL under Contracts AF 19(604)-5906 and AF 19(628)-462; 2) NASA under Contract NAS 5-3656.

I would like to acknowledge stimulating and helpful discussions with Lee Tepley, J. Cladis, R, Landshoff, and M. Walt at Lockheed and with R.A. Helliwell and D.L. Carpenter at Stanford.

\section{References}

Akasofu, S., and S. Chapman, The ring current, geomagnetic disturbance, and the Van Allen radiation belts, J. Geophys. Res., 66, 1321-1350, 1961.

Akasofu, S., J.C. Cain, and S. Chapman, The magnetic field of a model radiation belt, numerically computed, J. Geophys. Res., 66, 4013-4026, 1961.

Apel, J.R., Ceomagnetic field perturbations due to trapped particles, M.S. Thesis, Physics Dept., Univ. of Md., 1961.

Apel, J.R., S.F. Singer, and R.C. Wentworth, Effects of trapped particles on the geomagnetic field, Advances in Geophysics IX, Academic Press, New York (ed. H.F. Landsberg), 1962.

Benioff, H., Observations of geomagnetic fluctuations in the period range 0.3 to 120 seconds, J. Geophys. Res., 65, 1413-1422.

Campbell, W.H., Regular oscillations about $1 \mathrm{cps}$, Review paper presented at the Symposium on Ultra Low Frequency Electromagnetic Fields, Boulder, Colorado, August 17-27, 1964, to be presented in Radio Science.

Carpenter, D.L., Whistler evidence of a 'knee' in the magnetospheric ionization density profile, $J$. Geophys. Res., 68, 1675-1682, 1963.

Cornwall, J.M., Cyclotron instabilities and electromagnetic emission in the Ultra Low Frequency and Very Low Frequency ranges, J. Geophys. Res., 70, 61-69, 1965. 
Dawson, J.M, and I.B. Bernstein, Hydromagnetic instabilities caused by runaway electron, Controlled Thermonuclear Conf., Washington, D.C., TID 7558, p. 360, 1958.

Dessler, A.J., and E.N. Parker, Hydromagnetic theory of geomagnetic storms, J. Geophys. Res., 64, 22392252, 1959.

Dessler, A.J., Geomagnetism, Satellite Enviroment Handbook, Stanford University Press, Stanford, California (F.S. Johnson, ed.), 1965.

Duffus, H.J., P.W. Nasmyth, J.A. Shand, and C.S. Wright, Subaudible geomagnetic fluctuations, Nature, 181, 1258-1259, 1958.

Gendrin, R., Sur une theorie des pulsations rapides structurees, calcul des frequences observees, Compt. Rend., 256, 4487-4490, 1963a.

Gendrin, R., Sur une theorie des pulsations rapides structurees, caicul des intensites des oscillations observees, Compt Rend., 256, 4707-4710, 1963b.

Gendrin, R., Sur une theorie des pulsations rapides structurees, du champ magnetique terrestre, Ann. Geophys., 19(3), 197-214, 1963c.

Gendrin, R., and R. Stefant, Analyse de frequence des oscillations en Perls, Compt. Rend., 255, 752-754, 1962a.

Gendrin, R., and R. Stefant, Magnetic records between 0.2 and 30 cycles per second, communication presented at the Agard conference on propagation of radio frequencies below $300 \mathrm{Kc} / \mathrm{s}$, Munich, Germany, September 1962b.

Heacock, R.R., Notes on pearl-type micropulsations, J. Geophys. Res., 68, 589-591, 1963.

Heacock, R.R., and V.P. Hessler, Pearl-type telluric current micropulsations at College, J. Geophys. Res., 67, 3985-3996, 1962.

Jacobs, J.A., and T. Watanabe, Propagation of hydromagnetic waves in the lower exosphere and the origin of short period geomagnetic pulsations, J. Atmospheric Terrest. Phys., 24, 413-434, 1962.

Jacobs, J.A., and T. Watanabe, Micropulsations of the earth's electromagnetic field in the frequency range 0.1-10 cps, prepared for Commission IV, XIVth General Assembly URSI, Tokyo, Japan, September 1963a.

Jacobs, J.A., and T. Watanabe, Trapped charged particles as the origin of short-period geomagnetic pulsations, Planetary Space Sci., 11, 869-878, 1963b.

Jacobs, J.A., and T. Watanabe, Micropulsation whistlers, J. Atmos. and Terr. Phys., 26, 825-829, $1964 \mathrm{a}$.

Jacobs, J.A., and T. Watanabe, Hydromagnetic whistlers, presented at the Symposium on Ultra Low Frequency Electromagnetic Fields, Boulder, Colorado, August 17-27, 1964, to be published in Radio Science (b).

Jacobs, J.A., and T. Watanabe, Amplification of hydromagnetic waves in the magnetosphere by a cyclotron instability with applications to the theory of hydromagnetic whistlers, Geo-Astrophysics Laboratories, Boeing Scientific Research Laboratories, Seattle, Washington 98124, January 1965.

Liemohn, H.B., and F.L. Scarf, Whistler determination of electron energy and density distributions in the magnetosphere, J.. Geophys. Res., 69, 883-904, 1964.

Lokken, J.E., J.A. Shand, and C.S. Wright, Some characteristics of electromagnetic background signals in the vicinity of one cycle per second, J. Geophys. Res., 68, 789-794, 1963.

Mainstone, J.S., and R.W. E. McNicol, Screamer activity and Pearl pulsations, paper presented at the International conference on the ionosphere, London, July 1962.

Matveea, E.T., and V.A. Troitskaya, General regularities of the PP type oscillation, presented at the 13 th General Assembly of IUGG, Berkeley, August 1963.

Mead, G.D., Deformation of the geomagnetic field by the solar wind, J. Geophys. Res., 69, 1181-1195, 1964.

Ness, N.F., The earth's magnetic tail, presented at the Second Benedum Conference on Earth Magnet- 
ism, University of Pittsburgh, Pittsburgh, Pensylvania, November 23, 1964. Submitted to J. Geophys. Res.

Obayashi, T., The acceleration of particles in the outer atmosphere, J. Ceomag. Geoelect., 10, 151-152, 1959.

Obayashi, T., Hydromagnetic whistlers, presented at the Symposium on Ultra Low Frequency Electromagnetic Fields, Boulder, Colorado, August 17-27, 1964, J. Geophys. Res., 70, 1069-1078, 1965.

O'Brien, B.J., A large diurnal variation of the geomagnetically trapped radiation, J. Geophys. Res., 68, 989-995, 1963.

Piddington, J.H., Geomagnetic storm theory, J. Geophys. Res., 65, 93-105, 1960.

Singer, S.F., A new model of magnetic storms and aurorae, Trans. Am. Geophys. Union, 38, 175-190, 1957.

Smith, E.J., A comparison of Explorer VI and Explorer X magnetometer data, J. Ceophys. Res., 67, 2045-2049, 1962.

Stix, T.H., The theory of plasma waves, McGraw-Hill Book Company, Inc., 1962.

Storey, L.R. O., An investigation of whistling atmospherics, Phil. Trans. Roy. Soc., A 246, 113-141, 1953.

Tepley, L.R., Observations of hydromagnetic emissions, I. Geophys. Res., 66, 1651-1658, 1961.

Tepley, L.R., Structure and attenuation of hydromagnetic emissions, Vol. 1, Sci. Rept. 1 (contract AF 19(604)-5906, Electronic Research Directorate, Air Research and Development Command), April 6, 1962.

Tepley, L.R., Low-latitude observations of fine-structured hydromagnetic emissions, J. Geophys. Res., 69, 2273-2290, 1964.

Tepley, L.R., Regular oscillations near $1 \mathrm{cps}$ observed at middle and low latitudes, Review paper presented at the Symposium on Ultra Low Frequency Electromagnetic Fields, Boulder, Colorado, August 17-27, 1964, to be published in Radio Science.

Tepley, L.R., Recent investigations of hydromagnetic emissions, Part I: Experimental observations, Review paper presented at the Second Benedum Symposium on Earth Magnetism, University of Pittsburgh, Pittsburgh, Pennsylvania, November 23-25, 1964, in Journal of Geomagnetism and Geoeletricity, this insue, 1966.

Tepley, L.R., and R.C. Wentworth, Structure and attenuation of hydromagnetic emissions, Vol. 2, Sci. Rept. 1 (contract AF 19(604)-5906, Electronic Research Directorate, Air Research and Development Command), April 6, 1962a.

Tepley, L.R., and R.C. Wentworth, Hydromagnetic emissions, X-ray bursts, and electron bunches, Part I: Experimental results, J. Geophys. Res., 67, 3317-3333, 1962b.

Tepley, L.R., and R.C. Wentworth, Hydromagnetic emissions associated with the magnetic storm of September 30, 1961, J. Geophys. Res., 68, 3733-3737, 1963.

Tepley, L.R., R.C. Wentworth, and K.D. Amundsen, Sub ELF magnetic fluctuations, Vol. I : Frequencytime characteristics of hydromagnetic emissions, Final report, contract AF 19(628)-462 for Upper Atmosphere Physics Lacoratory, Air Force Cambridge Research Laboratories, Office of Aerospace Research, 1964.

Tepley, L.R., and R.C. Wentworth, Cyclotron excitation of hydromagnetic emissions, presented at the Symposium on Ultra Low Frequency Electromagnetic Fields, Boulder, Colorado, August 17-27, 1964.

Troitskaya, V., Earth current installations at the stations of the USSR, Ann. IGY 4, 322-329, 1957.

Watanabe, T., Hydromagnetic oscillation of the outer ionosphere and geomagnetic pulsation, J. Geomag. Geoelect., 10, 195-202, 1959.

Wentworth, R.C., Evidence for maximum production of hydromagnetic emissions above the afternoon hemisphere of the earth, Part 1: Extrapolation to the base of the exosphere, J. Ceophys. Res., 69, 2689-2698, 1964. 
Wentworth, R.C., and L.R. Tepley, Hydromagnetic emissions, X-ray bursts, and electron bunches, Part II : Theoretical interpretation, J. Geophys. Res., 67, 3335-3343, 1962.

Williams,-P.J., and W.F. Palmer, Distortions in the radiation cavity as measured by an 1100 -kilometer polar orbiting satellite, J. Geophys. Res., 70, 557-567, 1965.

Yanagihara, K., Some characteristics of geomagnetic pulsation pt and accompanied oscillation spt, J. Geomag. Geoelec., 10, 172-176, 1959.

Yanagihara, K., Geomagnetic micropulsations with periods from 0.03 to 10 seconds in the auroral zones with special reference to conjugate-point studies, I. Geophys. Res., 68, 3383-3397, 1963. 